\title{
A DNA-based vaccine protects against Crimean-Congo haemorrhagic fever virus disease in a Cynomolgus macaque model
}

\author{
David W. Hawman', Gustaf Ahlén ${ }^{2,6}$, K. Sofia Appelberg ${ }^{3,6}$, Kimberly Meade-White', \\ Patrick W. Hanley', Dana Scott', Vanessa Monteil $\oplus^{2}$, Stephanie Devignot $\circledast^{4}$, Atsushi Okumura', \\ Friedemann Weber ${ }^{\circledR}{ }^{4}$, Heinz Feldmann ${ }^{1,6}$, Matti Sällberg ${ }^{2,6}$ and Ali Mirazimi ${ }^{2,3,5,6 \bowtie}$
}

\begin{abstract}
There is currently no specific prophylaxis or vaccine against Crimean-Congo haemorrhagic fever virus (CCHFV). Crimean-Congo haemorrhagic fever (CCHF) is a severe febrile illness transmitted by Hyalomma ticks in endemic areas, handling of infected livestock or care of infected patients. We report here the successful protection against CCHFV-mediated disease in a non-human primate disease model. Cynomolgus macaques were vaccinated with a DNA-based vaccine using invivo electroporation-assisted delivery. The vaccine contained two plasmids encoding the glycoprotein precursor (GPC) and the nucleoprotein (NP) of CCHFV. Animals received three vaccinations and we recorded potent antibody and $T$ cell responses after vaccination. While all sham-vaccinated animals developed viraemia, high tissue viral loads and CCHF-induced disease, the NP + GPC vaccinated animals were significantly protected. In conclusion, this is evidence of a vaccine that can protect against CCHFV-induced disease in a non-human primate model. This supports clinical development of the vaccine to protect groups at risk for contracting the infection.
\end{abstract}

C rimean-Congo haemorrhagic fever (CCHF) is a severe febrile illness caused by the Crimean-Congo haemorrhagic fever virus (CCHFV), a negative-sense RNA virus in the Nairoviridae family of the Bunyavirales order. Aligning with the wide geographic distribution of its Hyalomma species tick vector and reservoir, cases of CCHF are reported throughout Eastern Europe, Africa, the Middle East and parts of Asia ${ }^{1}$. In addition, recent cases of CCHF have been reported in Spain ${ }^{2}$ and global climate change is likely to lead to expansion of the range of the Hyalomma tick leading to introduction of CCHFV into new areas. CCHFV-exposure typically occurs through tick bites, handling of infected livestock or during the treatment of infected patients in the health care setting. CCHF begins as a non-specific febrile illness that can rapidly progress to severe haemorrhagic manifestations ${ }^{3}$. Case fatality rates vary among regions but typically range between 5 and 30\% (ref. ${ }^{3}$ ). Currently, treatment options for CCHFV are lacking. While ribavirin is recommended by the World Health Organization $^{4}$, clinical data from human cases ${ }^{5}$ and animal models ${ }^{6-8}$ provide conflicting evidence on the benefit of ribavirin for treatment of CCHFV and indicate better treatments are needed. The World Health Organization has put CCHFV on its blueprint list to highlight the need to develop antivirals and vaccines (https:// www.who.int/blueprint/).

A safe, effective vaccine is critically needed for at-risk populations such as health care workers, rural inhabitants and abattoir workers. As CCHFV infection of livestock is apparently asymptomatic ${ }^{9}$, farmers and abattoir workers may be unknowingly infected with CCHFV during the handling and care of livestock. Similarly, tick bites by Hyalomma ticks may not be fully appreciated as a risk factor for CCHFV by rural inhabitants in endemic areas. Thus, the early clinical signs of CCHF, fever, headache and myalgia ${ }^{3}$, may not be recognized as the early stages of CCHF. This is problematic as clinical evidence for the efficacy of ribavirin in treatment of CCHF indicates that ribavirin must be given early after symptom onset to offer clinical benefit ${ }^{5,10}$. Consequently, by the time many patients present at health care facilities, they are exhibiting the more severe signs of CCHF, when treatment is limited to supportive care. Therefore, vaccination of at-risk populations against CCHFV probably represents the most effective therapeutic intervention to limit CCHFV-induced morbidity and mortality. While an inactivated preparation of CCHFV grown in mouse brains has been used in eastern Europe ${ }^{11,12}$, practical and safety considerations will probably prevent this vaccine from achieving wide-spread distribution. Several vaccine platforms have been evaluated in mouse models including modified vaccinia Ankara-based, vesicular stomatitis virus-based, human adenovirus-based, subunit-based, DNA-based, virus-like particle-based and transgenic plant-based vaccines $^{13-21}$. We have recently developed a non-human primate model of $\mathrm{CCHF}^{22,23}$ in which cynomolgus macaques inoculated with the clinical isolate of CCHFV strain Hoti recapitulate many aspects of human CCHF. So far, no vaccine candidates have been evaluated in this immunocompetent animal disease model and evaluation of vaccine candidates in this unique model will provide important preclinical data on the safety, immunogenicity and protection of potential vaccine candidates for CCHFV.

DNA-based vaccines developed by our group and others have demonstrated efficacy against CCHFV in lethal mouse challenge models ${ }^{15,19}$ and DNA-based vaccines have a favourable safety profile 
a b

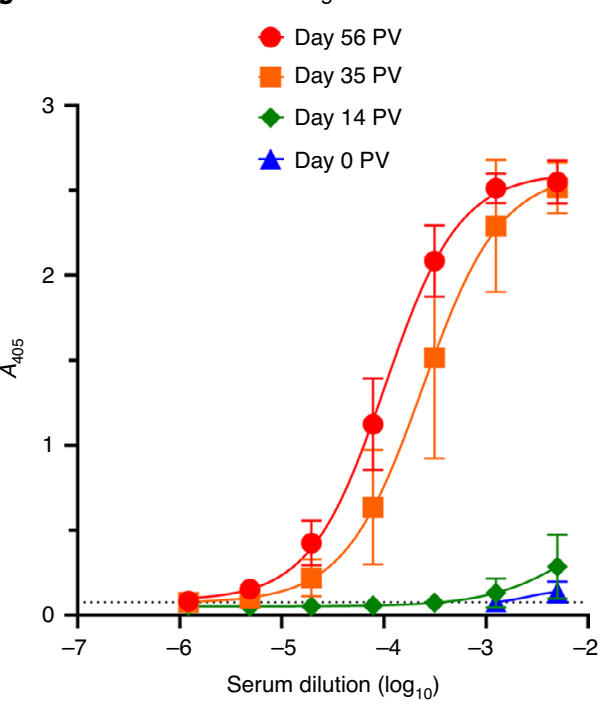

c

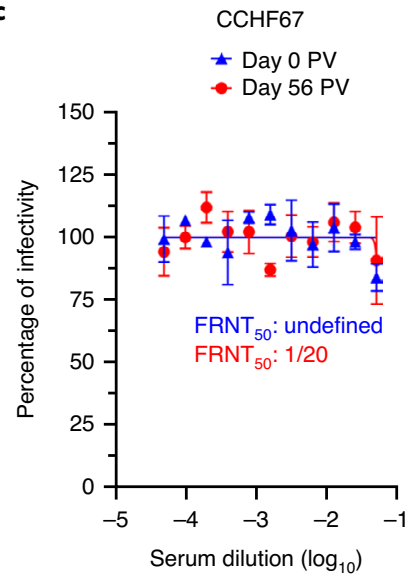

CCHF70

^ Day 0 PV

- Day 56 PV

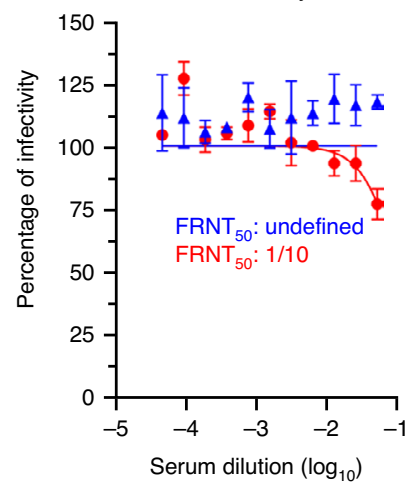

Day 56 PV

- Day 35 PV

- Day 14 PV

^ Day O PV

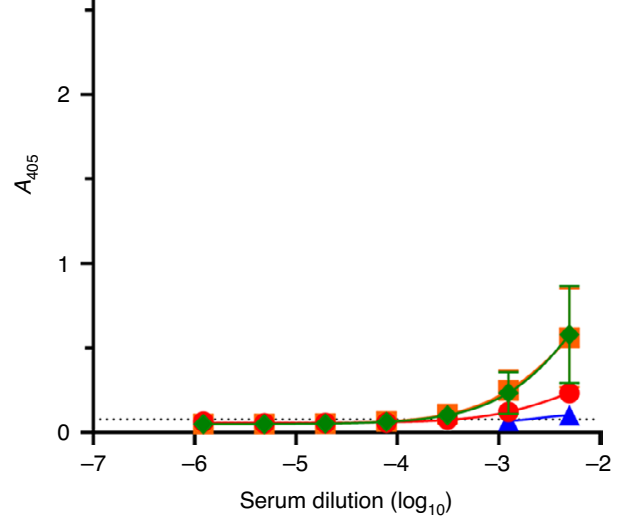

CCHF68

- Day 0 PV

- Day 56 PV

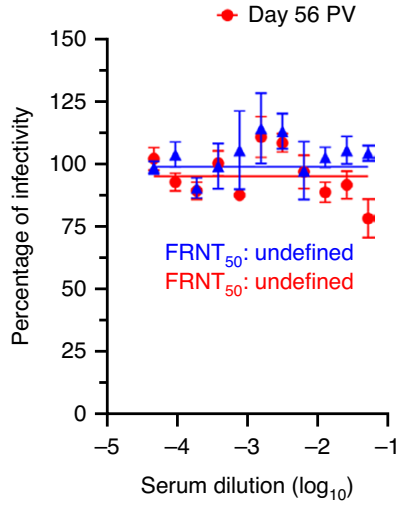

CCHF71

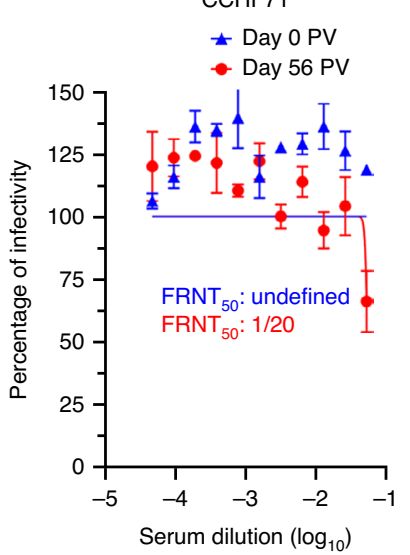

$\lg G$

Day 56 PV

Day 35 PV

Day 14 PV

Day 0 PV

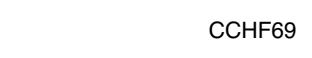

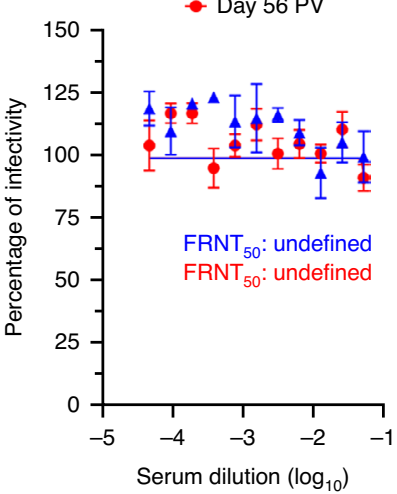

CCHF72

\ Day 0 PV

- Day 56 PV

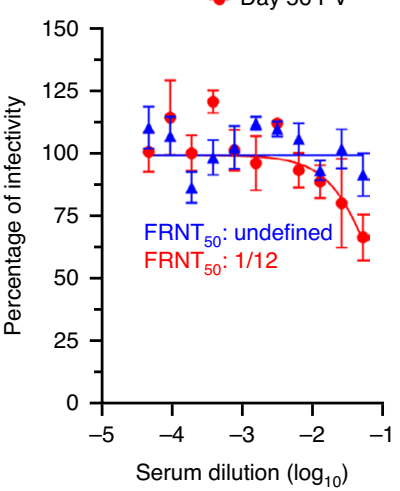

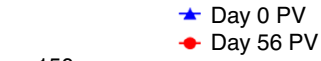

Fig. 1 | NP + GPC vaccination induces robust antibody responses to CCHFV. a,b, At indicated timepoints, CCHFV-specific IgM (a) and IgG (b) antibody responses in the serum were measured by whole-virion ELISA. Data are shown as the mean plus the standard deviation of duplicate measurements. Connecting lines were derived from unconstrained non-linear regression. Dashed line indicates average absorbance of background wells receiving no serum. $A_{405}$, absorbance at $405 \mathrm{~nm}$. c, FRNT was performed to measure neutralizing activity of day -7 serum from NP + GPC vaccinated animals. Individual neutralization curves for each NP + GPC vaccinated animals are shown. Non-linear regression with a top constraint of 100 and bottom constraint of 0 was calculated and the $\mathrm{FRNT}_{50}$ determined. Data are shown as mean plus standard error of measurement from triplicate technical replicates. $n=6$ animals per group.

owing to their non-replicating nature ${ }^{24}$. Hence, DNA-based vaccines represent a promising platform for development of CCHFV vaccines. We report here evaluation of a DNA-based vaccine con- sisting of plasmid-expressed CCHFV strain Hoti nucleoprotein (NP) and the CCHFV strain Hoti M-segment open-reading frame encoding the viral glycoproteins (GPC) in the cynomolgus macaque 


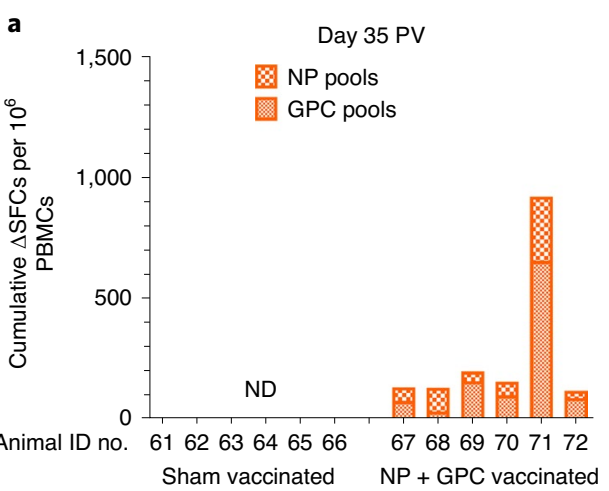

C

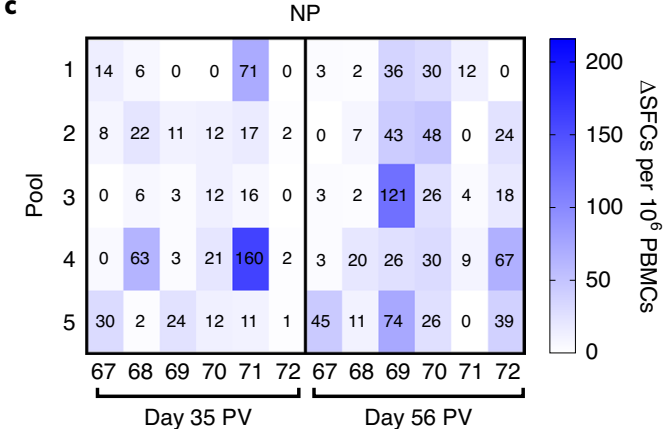

b

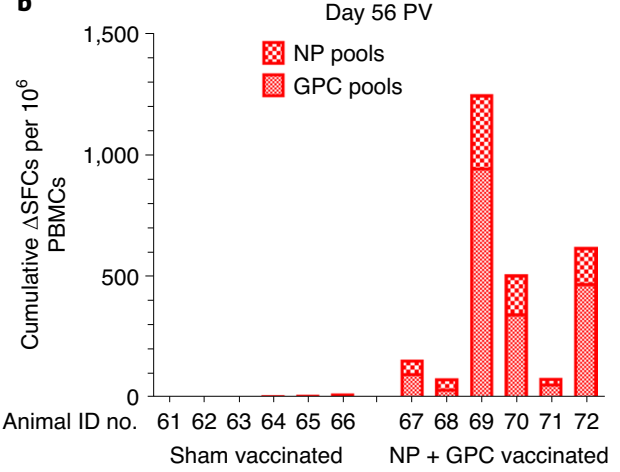

d

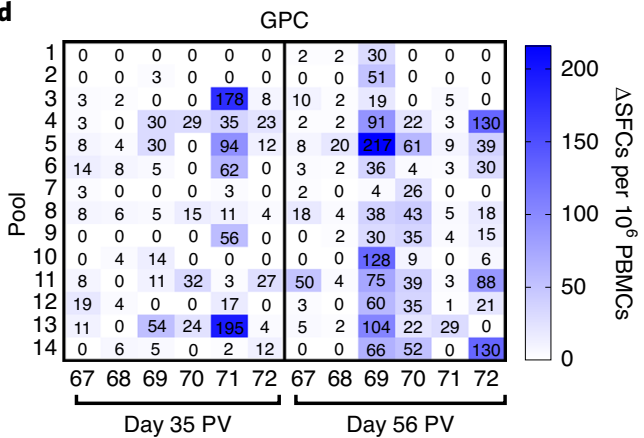

Fig. 2 | NP + GPC vaccination induces CCHFV-specific IFN- $\boldsymbol{\gamma}$ T cell responses. a,b, Cryopreserved PBMCs from day 35 (a) or day 56 PV (b) were stimulated with pooled overlapping peptides derived from the CCHFV GPC or NP at $1 \mu \mathrm{gg} \mathrm{ml}^{-1}$ per peptide. Background was ascertained by wells stimulated with DMSO vehicle alone. After $20 \mathrm{~h}$, IFN- $\gamma$ producing cells were detected by ELISpot. SFCs were counted, background count subtracted from peptide-stimulated wells ( $\triangle$ SFCs) and normalized to $1 \times 10^{6}$ PBMCs. All measurements were performed in duplicate for each animal. Data are shown as the sum of SFCs counted among all NP and GPC peptide pools. c,d, For NP + GPC vaccinated animals, the number of SFCs against each peptide pool for NP (c) and GPC (d) is shown. ND, not done.

model. Plasmid DNA was delivered by intramuscular injections followed by in vivo electroporation. Vaccination appeared well tolerated and elicited CCHFV-specific antibody and T cell responses. Such responses in vaccinated animals correlated with absent viraemia, substantially reduced viral burdens in all tissues evaluated and improved blood parameters on CCHFV challenge. Together, our data show that this vaccine provides significant protection against CCHFV in a non-human primate model.

\section{Results}

Vaccination of animals. We have previously demonstrated that interferon signalling-incompetent $\mathrm{IFNAR}^{-/}$mice immunized with DNA coding for the viral NP, Gn and Gc of CCHFV strain IbAr 10200 were protected against homologous lethal CCHFV challenge $^{19}$. The recent development of a non-human primate model for CCHF, based on CCHFV strain Hoti $^{22}$, enabled evaluation of this DNA-based vaccination approach in fully immunocompetent animals. Since our initial report on CCHFV infection of cynomolgus macaques, we have further developed the model using combined subcutaneous and intravenous inoculation ${ }^{23}$. Until now, infection of cynomolgus macaques has demonstrated that CCHFV infection results in a range of disease outcomes from mild-to-moderate-to-severe disease, similar to the range of disease seen in humans. Since the model is not uniformly lethal, we designed a study with a scheduled euthanasia at day 6 post-CCHFV infection to evaluate vaccine-mediated protection, similar to the rhesus macaque model for Middle-Eastern respiratory virus and severe acute respiratory syndrome coronavirus-2 (SARS-CoV-2) ${ }^{25-27}$ and similar to our study evaluating favipiravir in CCHFV-infected cynomolgus macaques $^{23}$. Twelve female Chinese-origin adult cyno- molgus macaques, Macaca fascicularis, were used for this study (Extended Data Fig. 1).

Six animals were vaccinated with two ubiquitin-antigen fusion plasmids, one encoding the CCHFV NP and the other the viral GPC, both derived from CCHFV strain Hoti. One milligram of each respective plasmid was delivered independently to opposing quadriceps. Six animals also received a sham vaccination of an equivalent dose of plasmid encoding ubiquitin to each quadricep followed by in vivo electroporation. Animals received three identical vaccinations separated by 3 -week intervals (Extended Data Fig. 1). Blood draws were taken before vaccination and 2 weeks after each vaccination (Extended Data Fig. 1). The electroporation delivery of the vaccine appeared well tolerated. No serious adverse events were observed, and the most common side-effect observed during clinical exams following vaccinations was firmness at the vaccination site that resolved without intervention; this induration is expected after vaccination. In one animal, a lesion developed at the vaccination site, probably due to pruritis, but this too resolved without intervention. At time of scheduled euthanasia following CCHFV challenge ( $27 \mathrm{~d}$ after last vaccination), histopathological analysis of the vaccination site indicated no pathology in four of the vaccinated animals. Six animals had minor, one had moderate and one had severe myonecrosis and loss with histiocytosis with intracellular pigment at the vaccination site (Extended Data Fig. 2). These histologic lesions were limited to the immediate site of vaccination and at no time postvaccination did any animals display clinical signs to indicate pain or discomfort associated with muscular lesions.

DNA-vaccination induces $B$ and $T$ cell responses to $\mathrm{CCHF}$ antigens. To evaluate the antibody response to the vaccine, 
serum was collected at time of first immunization (prebleed) and 2 weeks after each immunization thereafter (Extended Data Fig. 1) and CCHFV-specific IgM and IgG quantified by whole-virion enzyme-linked immunosorbent assay (ELISA). Although we detected CCHFV-specific IgM 2 weeks after the first immunization (day 14 postprime vaccination (PV)) (Fig. 1a), strong CCHFV-specific IgG responses were not detected until 2 weeks after the second immunization (day 35 PV) (Fig. 1b). We detected slight increases in CCHFV-specific IgG titre after the third immunization (day 56 PV) (Fig. 1b). As expected, sham-vaccinated animals had no change in ELISA signal between prebleed (day 0 PV) and day 56 PV (Extended Data Fig. 3). We also evaluated whether the CCHFV-specific antibody response we detected in NP + GPC vaccinated animals by ELISA was neutralizing. Neutralization capacity of heat-inactivated serum collected at day $0 \mathrm{PV}$ or on day $56 \mathrm{PV}$ was evaluated by focus reduction neutralization tests (FRNTs). Despite high levels of antibody detected by ELISA, day 56 PV serum demonstrated poor neutralization activity against infectious CCHFV (Fig. 1c). In light of the high ELISA titre but poor serum neutralizing activity, we performed a sandwich ELISA to specifically measure NP or Gc-specific responses. Consistent with the poor neutralizing activity, we did not detect significant increases in Gc-specific antibody responses between day 0 and 56 PV (Extended Data Fig. 4). In contrast, significant increases between day 0 and $56 \mathrm{PV}$ in NP-specific antibody responses were detected (Extended Data Fig. 4 ), suggesting the humoral response was largely directed against NP.

To evaluate $\mathrm{T}$ cell responses, we performed an interferon gamma (IFN- $\gamma$ ) ELISpot on cryopreserved peripheral blood mononuclear cells (PBMCs) collected 2 weeks after the second (day 35 $\mathrm{PV}$ ) and third vaccinations (day $56 \mathrm{PV}$ ) (Fig. 2). Compared to sham-vaccinated animals, all NP + GPC vaccinated animals had detectable in vitro recall responses measured as IFN- $\gamma$-producing spot forming cells (SFCs) to CCHFV-peptides, indicating the priming of CCHFV-specific $\mathrm{T}$ cell responses by the vaccination (Fig. $2 a, b)$. Recall responses against both NP and GPC-derived peptide pools were detected in all animals. Responses were equally distributed across NP (Fig. 2c). Across the GPC, we did not identify an immunodominant peptide pool although minimal responses were directed against N-terminal pool nos. 1 or 2 or pool no. 7 (Fig. 2d). Together, our ELISpot data demonstrate that NP + GPC vaccination induced CCHFV-specific IFN- $\gamma$ recall responses against both vaccine antigens.

NP+ GPC DNA-vaccination protects against CCHFV-induced disease. To evaluate the protection afforded by NP + GPC vaccination, on day 0 relative to CCHFV challenge (postinfection (PI)) and day $63 \mathrm{PV}$, sham-vaccinated and NP + GPC vaccinated animals were challenged with 100,000 median tissue culture infectious dose $\left(\mathrm{TCID}_{50}\right)$ of CCHFV strain Hoti via simultaneous subcutaneous and intravenous injections ${ }^{22}$. Although this model is not uniformly lethal, inoculation of naïve animals via these routes results in consistent development of viraemia, blood haematology and chemistry perturbations as well as significant viral burdens in a variety of tissues following CCHFV infection ${ }^{22}$. Animals were comprehensively scored daily and NP + GPC vaccinated animals had significantly reduced clinical signs of disease on day +6 (Fig. $3 a$ and Extended Data Fig. 5a). Clinical exams on days $0,3,5$ and 6 PI were performed and blood collected for evaluation of blood chemistry and haematology changes between groups. When compared to baseline values obtained on day 0 , we found that sham-vaccinated animals had significantly reduced platelets, total protein and albumin levels along with significantly elevated aspartate aminotransferase (AST) values at days 3, 5 and 6 PI (Fig. 3b-e and Extended Data Fig. 5b-e). In contrast, these values in NP + GPC vaccinated animals remained largely unchanged from day 0 with slight decreases in albumin on days 3 and 6 PI and even significantly elevated plate-
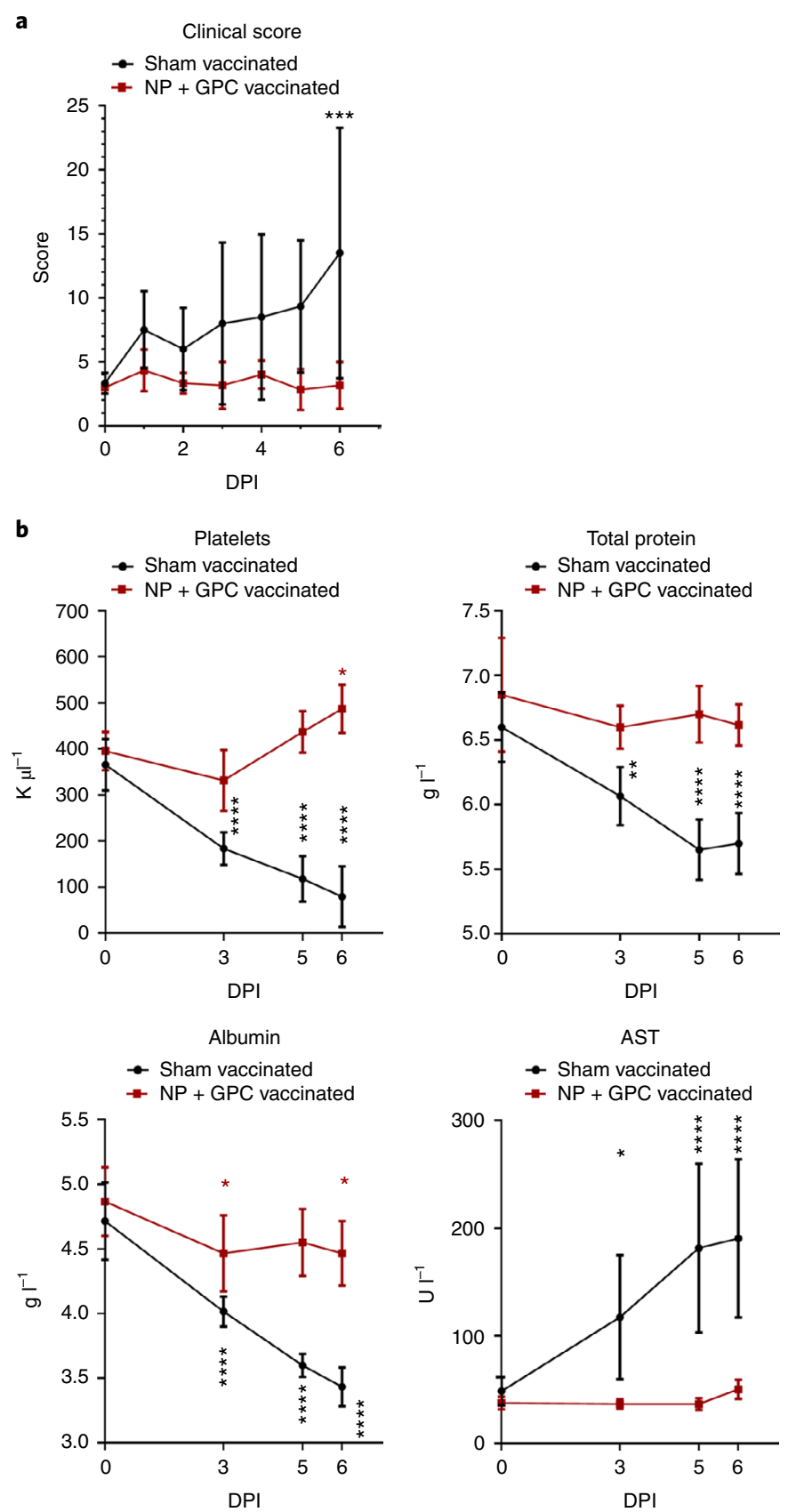

Fig. 3 | NP + GPC vaccination improves clinical scores and blood chemistry following CCHFV challenge. a, Animals were comprehensively scored for evaluation of overt clinical disease and cumulative clinical scores are shown. Data are shown as mean plus standard deviation. Statistical comparison was performed by two-way analysis of variance (ANOVA) with Sidak's multiple comparison test at each timepoint. Day $6 \mathrm{PI},{ }^{\star \star \star} P=0.0003$. b, At indicated timepoints platelets were enumerated in EDTA-treated whole-blood (normal range 300,000$500,000 \mu^{-1}$ ) and serum was evaluated for total protein (normal range $\left.7.5 \mathrm{~g} \mathrm{dl}^{-1} \pm 0.57\right)$, albumin $\left(4.2 \mathrm{~g} \mathrm{dl}^{-1} \pm 0.28\right)$ and AST (48.2 IU per \pm \pm 36 ). Data are shown as mean plus standard deviation. Statistical comparison was performed between baseline values on day 0 and the indicated timepoint. $n=6$ animals per group. Platelets, ${ }^{\star \star \star \star} P<0.0001,{ }^{\star} P=0.0129$. Total protein, ${ }^{\star \star \star \star} P<0.0001,{ }^{\star \star} P=0.0027$. Albumin, ${ }^{\star \star \star \star} P<0.0001$, ${ }^{\star} P=0.0131$. AST, ${ }^{\star \star \star \star} P<0.0001,{ }^{\star} P=0.0280$. $P$ values calculated with a two-tailed two-way ANOVA with Sidak's multiple comparison test. DPI, days post infection. 
a

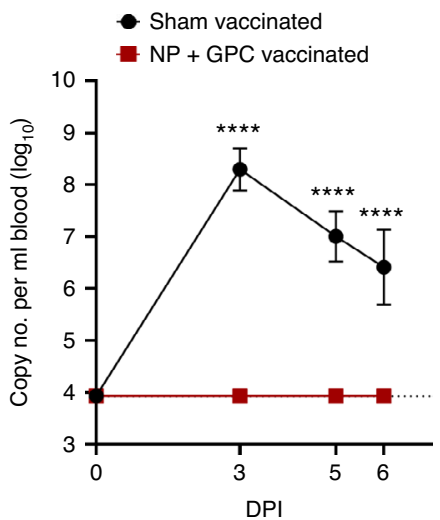

b

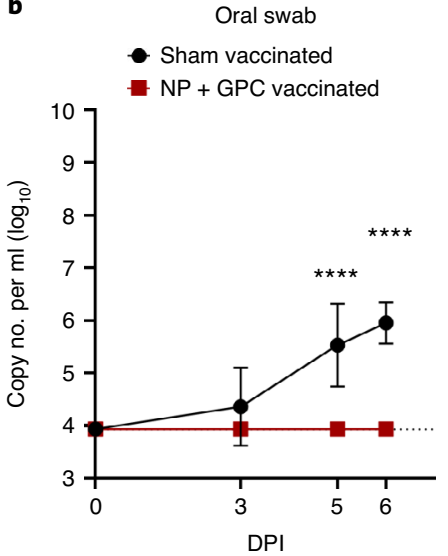

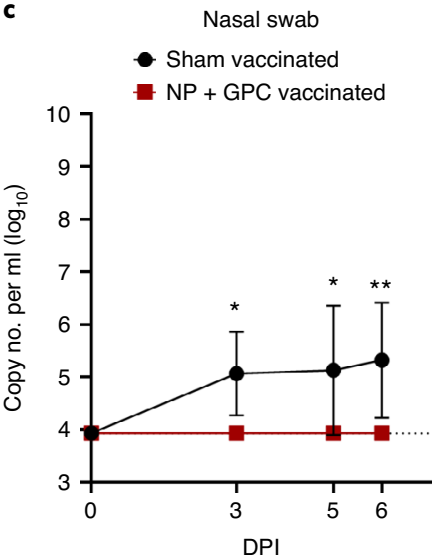

Fig. 4 | NP + GPC vaccination prevents viraemia and viral shedding. a-c, Viral genomes were quantified by RT-qPCR at the indicated timepoints. Data are shown as mean plus standard deviation. The dashed line indicates limit of detection. Viraemia (a) and oral swab (b), ${ }^{\star \star \star \star} P<0.0001$. Nasal swab (c), ${ }^{\star} P=0.0169(3 \mathrm{DPI})$ or $0.0109(5 \mathrm{DPI}),{ }^{\star \star} P=0.0025$. $P$ values calculated with a two-tailed two-way ANOVA with Sidak's multiple comparison test. $n=6$ animals per group.

lets on day 6 (Fig. 5b-e and Extended Data Fig. 5b-e), although these values remained within normal ranges for NP + GPC vaccinated animals. These data demonstrate that NP + GPC vaccination prevents changes in blood chemistry that are often associated with poor outcome in human CCHF cases $^{3,28}$. Other blood chemistry and haematology parameters were largely similar between groups. Complete blood chemistry and haematology profiles are provided in the Supplementary Information.

DNA-vaccination prevents viraemia, viral shedding and reduces viral RNA burdens in multiple tissues. To determine shedding of virus following CCHFV challenge, we evaluated viral RNA burdens in blood and oral and nasal swabs on days 0, 3, 5 and 6 PI (Fig. 4). We found that NP + GPC vaccinated animals had no detectable viral RNA in the blood at any timepoint evaluated (Fig. 4a). In contrast, sham-vaccinated animals had significant levels of viral RNA at 3, 5 and 6 days PI (Fig. 4a). Similarly, NP + GPC vaccinated animals had no detectable viral RNA in the oral or nasal cavities whereas most sham-vaccinated animals had detectable viral RNA in these samples (Fig. 4b,c). Cumulatively, NP+ GPC vaccinated animals had no detectable viral RNA in the blood, oral cavity or nasal cavity at any timepoint evaluated suggesting NP + GPC vaccinated animals exhibited robust control of the CCHFV challenge.

Since our study was not powered to detect differences in survival between sham- and NP + GPC vaccinated groups, a scheduled necropsy was performed on day 6 PI to evaluate viral burdens and histopathology in a variety of tissues. We found that NP+GPC vaccination significantly reduced viral RNA burdens in all tissues evaluated (Fig. 5). In key organs such as the liver, kidney, lung and adrenal gland, NP + GPC vaccination reduced viral RNA to undetectable levels in most vaccinated animals (Fig. 5a). NP+GPC vaccinated animals also had significantly reduced viral burdens compared to sham-vaccinated animals at the injection site of the subcutaneous CCHFV inoculation (Fig. 5a). Four of six NP+ GPC vaccinated animals had no detectable CCHFV RNA at the injection site suggesting these animals effectively cleared CCHFV RNA from the site of inoculation within $6 \mathrm{~d}$ PI. We also collected the spleen and lymph nodes to evaluate CCHFV viral burdens in lymphoid tissues. In these tissues, NP + GPC vaccination significantly reduced CCHFV RNA burdens compared to sham-vaccinated animals, with a greater than 100 -fold reduction in viral burden in all tissues (Fig. 5b). To further evaluate CCHFV burdens in the liver, we performed immunohistochemistry to detect CCHFV NP anti- gen. All six sham-vaccinated animals but no NP+ GPC vaccinated animals had detectable CCHFV antigen in the liver (Fig. 5c-f), consistent with our quantitative reverse transcription PCR (RTqPCR) data. Last, when we evaluated serum antibody responses after challenge on day 6 PI, we found no significant increases in CCHFV-specific titre (Extended Data Fig. 6) indicating no anamnestic antibody response, at least to day 6 PI. These data are consistent with the significantly reduced viral loads in multiple tissues and substantial vaccine-mediated protection against CCHFV challenge. Cumulatively, our data demonstrated that NP + GPC vaccination significantly reduced CCHFV viraemia, viral shedding and viral burdens in a multitude of tissues.

\section{Discussion}

There is a critical need for vaccines against CCHFV particularly for populations at risk of exposure to CCHFV. We report here a DNA-based vaccine that elicits humoral and cellular immunity against CCHFV. These responses provided significant protection against $\mathrm{CCHFV}$ challenge. Although the cynomolgus macaque model of CCHF is not uniformly lethal and we were unable to power the study to demonstrate improvements in survival, NP + GPC vaccination improved several key parameters that are associated with poor outcome in human CCHF cases including viraemia, platelet count and liver enzymes ${ }^{29,30}$. High viral loads are often associated with a poor outcome in human CCHF cases $^{29-31}$ and NP + GPC vaccinated animals had no detectable viraemia at any timepoint evaluated, even when using a sensitive RT-qPCR assay. In addition to absent CCHFV viraemia, NP + GPC vaccinated animals had significantly reduced CCHFV loads in a multitude of tissues.

Thrombocytopenia is a common clinical feature of CCHF and low platelet counts typically correlate with prolonged clotting times and poor outcome $e^{28,32,33}$. Further, decreases in total protein and albumin have also been noted in severe CCHFV cases ${ }^{28}$. While sham-vaccinated animals showed significant declines in platelet counts, total protein and albumin following CCHFV infection, $\mathrm{NP}+\mathrm{GPC}$ vaccinated animals exhibited no such decline. Although we did not measure clotting times in our study, platelet counts of $\mathrm{NP}+\mathrm{GPC}$ vaccinated animals remained within normal ranges suggesting vaccination protected against dysregulated clotting function. Similarly, AST levels, often elevated in severe and fatal human cases of $\mathrm{CCHF}^{31,33}$, were significantly elevated in sham-vaccinated but not $\mathrm{NP}+\mathrm{GPC}$ vaccinated animals. These data indicate that the immune responses induced by $\mathrm{NP}+\mathrm{GPC}$ vaccination were highly effective in 


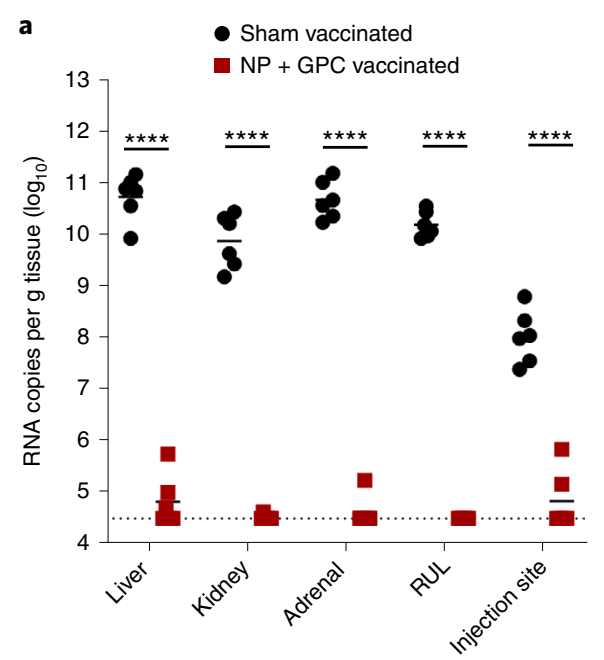

c

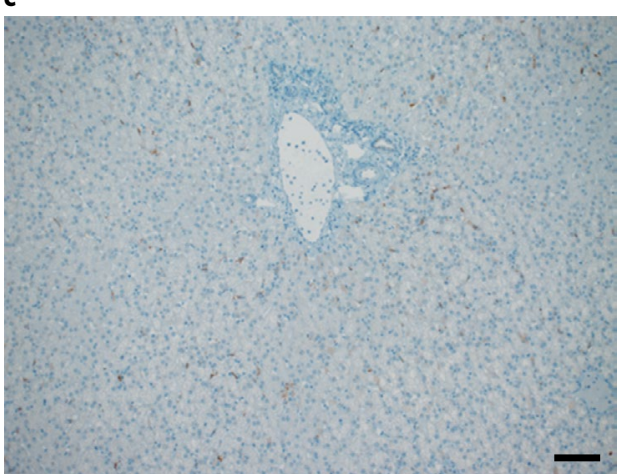

d

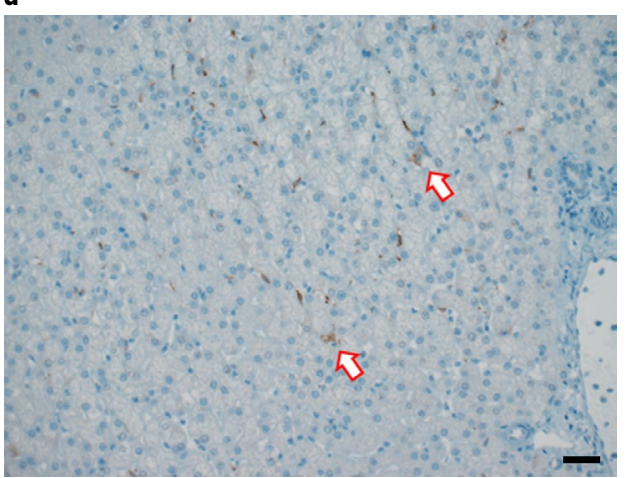

b
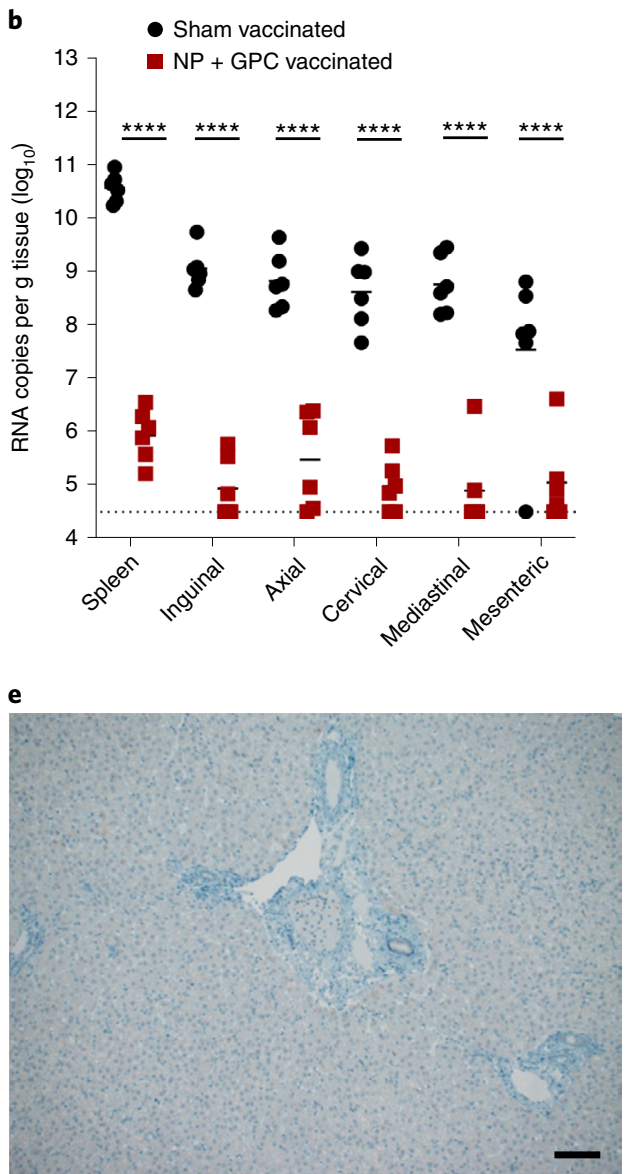

f

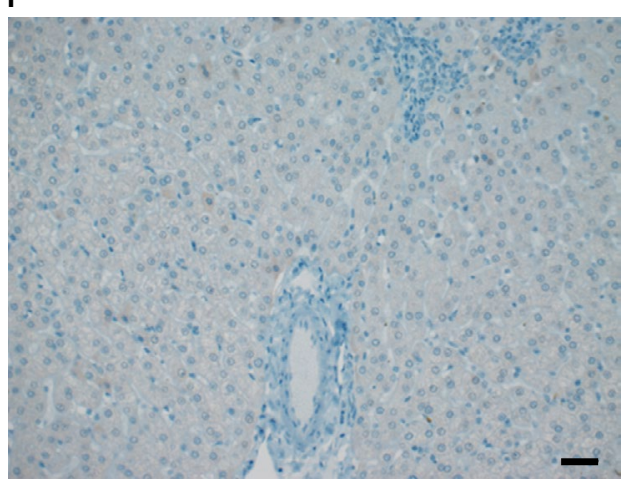

Fig. 5 | NP + GPC vaccination reduces viral burden in multiple tissues. a,b, At +6 DPI, a scheduled necropsy was performed and viral genomes quantified in the indicated organs or tissues (a) or lymphoid tissues (b) by RT-qPCR. Individual values are shown, bar indicates mean and dashed line indicates limit of detection. ${ }^{\star \star \star \star} P<0.0001$. $P$ values calculated with a two-tailed two-way ANOVA with Sidak's multiple comparison test. RUL, right upper lung. $\mathbf{c}-\mathbf{f}$, Immunohistochemistry to detect CCHFV antigen in the liver was performed and representative images of sham-vaccinated (c,d) and NP + GPC vaccinated $(\mathbf{e}, \mathbf{f})$ animals are shown at $\times 100$ (c and $\mathbf{e}$ ) or $\times 200$ (d and f) magnification. Arrows indicate antigen positive cells. Six sections from six animals per group were stained for CCHFV antigen and representative images from one animal per group shown. Scale bars, $50 \mu \mathrm{m}$ (c and $\mathbf{e}) ; 100 \mu \mathrm{m}$ (d and $\mathbf{f})$.

controlling the CCHFV challenge, reducing viral loads in multiple tissues and preventing perturbations to blood chemistry and haematology that are often associated with poor outcome in humans.

It has been previously shown that both $\mathrm{B}$ and $\mathrm{T}$ cell responses contribute to protection conferred by a modified vaccinia virus vaccine for $\mathrm{CCHFV}^{13,34}$. Our data demonstrate that $\mathrm{NP}+\mathrm{GPC}$ vaccination induced a robust but non-neutralizing antibody response, indicating neutralizing antibody responses, in the context of this vaccine, are dispensable for vaccine-mediated protection. Non-neutralizing antibodies can contribute to protection against $\mathrm{CCHFV}$ as non-neutralizing antibodies against $\mathrm{Gn}$ or
GP38 were protective when administered therapeutically in a lethal mouse model ${ }^{35,36}$. Further, a subunit vaccine induced neutralizing responses but failed to protect lethally infected mice ${ }^{17}$ indicating that responses beyond neutralizing antibodies may be required for vaccine-mediated protection from CCHFV. The role of $\mathrm{T}$ cells in vaccine-mediated protection from $\mathrm{CCHFV}$ is less clear. In studies evaluating a modified vaccinia Ankara virus expressing CCHFV antigens, both humoral and cellular immunity was required for protection $^{13,34}$. Cumulatively, our data indicate that all NP + GPC vaccinated animals developed CCHFV-specific antibody and IFN- $\gamma$ recall responses. 
We chose to vaccinate animals with plasmids expressing NP and GPC. Although GPC encodes the glycoproteins Gn and Gc, which are the target of protective and neutralizing antibodies ${ }^{35,37}$, NP based vaccines can confer significant protection on their own ${ }^{14}$. However, this may depend on the vaccine platform ${ }^{38}$. Furthermore, the GPC of CCHFV exhibits the lowest sequence conservation among the viral segments with some strains differing by up to $15 \%$ in $\mathrm{Gn}$ and Gc at the amino acid level ${ }^{1,39}$. This sequence variation can lead to impairment of antibody-mediated neutralization ${ }^{37}$ and could lead to incomplete protection when the vaccine antigens are mismatched to the infecting virus strain. Thus, the greater sequence conservation of NP and potentially protective immune responses directed against this antigen may favour the inclusion of NP in CCHFV-vaccine preparations. DNA-immunization of mice with full length GPC only provided partial protection in lethally infected mice ${ }^{15}$ whereas mice immunized with the glycoproteins and NP were completely protected $^{19}$. While we cannot exclude the possibility that differences in vaccine delivery, mouse strain or challenge dose may contribute to these distinct outcomes, it suggests inclusion of NP may improve vaccine-mediated protection.

Last, observation of immunized animals together with our immunology and challenge data indicate in vivo electroporation of plasmid DNA was well tolerated and immunogenic. No serious adverse events were observed in any of the vaccinated animals. Vaccination site reactions were mild and histopathological analysis of the vaccination sites at time of necropsy indicated absent-to-mild pathology in most animals. These data are consistent with other preclinical data and human clinical trials demonstrating the safety and immunogenicity of in vivo electroporation to deliver DNA-based vaccine platforms ${ }^{40-42}$.

There are several important limitations of our study that will require further investigation. First, in many CCHFV endemic regions, the three-vaccination regimen evaluated here may be difficult to achieve in at-risk populations due to remoteness and lack of infrastructure. Vaccinations providing protection after one or two immunizations would certainly be preferred for these communities. Some CCHFV experimental vaccines have shown efficacy in type I IFN-deficient mice after a single-dose ${ }^{13,20,21}$. However, our immunogenicity data certainly indicate that the third vaccination may not be needed and the use of immunodeficient mice may not strictly predict efficacy in immunocompetent non-human primates and humans ${ }^{43,44}$. Second, our study design of performing a timed necropsy on day 6 PI was chosen to maximize readout parameters but it prevented us from evaluating the possibility of delayed disease development in sham and NP + GPC vaccinated animals. However, before euthanasia no NP+ GPC vaccinated animals exhibited any indications of worsening clinical disease or declining immunity to suggest viral escape from the host response and even naïve animals begin to recover around day $7 \mathrm{PI}^{22}$. Last, we did not determine the immune correlates of protection in our study. NP + GPC vaccinated animals developed both humoral and cellular immunity against $\mathrm{CCHFV}$ and it is possible that either or both mediated the protection we observed in this study. Similarly, it is unclear if both NP and GPC vaccine-expressed antigens are needed for the observed protection. A vaccine containing just one antigen would simplify production and clinical evaluation and further studies are planned to evaluate vaccination with just NP or GPC alone.

In summary, our data demonstrate that a DNA-based vaccine delivered by in vivo electroporation against CCHFV is well tolerated, immunogenic and protects against disease in a preclinical non-human primate model of CCHF. Vaccinated animals showed CCHFV-specific antibody and $\mathrm{T}$ cell responses and on CCHFV challenge showed improved clinical parameters, significantly reduced viraemia, viral shedding and viral burdens in several key tissues including the liver. Further studies are needed to evaluate prime/boost versus prime-only vaccination strategies, durability of immune responses and to determine the correlates of protection in this model. However, our data indicate that this NP+ GPC DNA-driven vaccine platform may be a safe and effective vaccine to prevent the morbidity and mortality caused by CCHFV. The current study supports that this vaccine can be advanced into human clinical trials.

\section{Methods}

Animals, biosafety and ethics. All infectious work with CCHFV and sample inactivation was performed in the maximum containment laboratory in accordance with standard operating procedures approved by the Rocky Mountain Laboratories Institutional Biosafety Committee, Division of Intramural Research, National Institute of Allergy and Infectious Diseases, National Institutes of Health (Hamilton). All animal work was performed in strict accordance with the recommendations described in the Guide for the Care and Use of Laboratory Animals of the Office of Animal Welfare, National Institutes of Health and the Animal Welfare Act of the US Department of Agriculture, in an Association for Assessment and Accreditation of Laboratory Animal Care International (AAALAC)-accredited facility. Twelve female cynomolgus macaques of Chinese origin, 3-4 years of age were used in this study. Animals were housed in adjoining individual primate cages that enabled social interaction, under controlled conditions of humidity, temperature and light (12-h light/12-h dark cycles). Food and water were available ad libitum. Animals were monitored at least twice daily (pre- and postinfection) and fed commercial monkey chow, treats and fruit twice a day by trained personnel. Environmental enrichment consisted of manipulanda, visual enrichment and audio enrichment.

Plasmids. To create the pVAX1-Ub universal fusion vector, the ubiquitin sequence was subcloned from plasmid pCMV_Ub_F1F2 (ref. ${ }^{45}$ ) into pVAX1 (Invitrogen), using NheI restriction enzyme. A Kozak sequence was added in $5^{\prime}$, and a linker with a KpnI recognition site was added before the ubiquitin stop codon to allow subsequent cloning of antigen sequence. Protein expression from this construct will result in $5^{\prime}$-ubiquitin-antigen fusion ${ }^{46}$ (Extended Data Fig. 6). The ubiquitin protein has the mutation G76A to prevent its cleavage from the fusion protein, and all pVAX1 plasmids are Kanamycin resistant. Complementary DNA sequences from CCHFV strain Hoti S and M segments (GenBank accession nos. MH483984 and MH483985) were codon-optimized for expression in human cells and cloned in a shuttle vector (BioCat). All inserts were fully sequenced. To create the plasmids used for immunization, the pVAX1-Ub vector was linearized by using restriction enzymes KpnI and NotI, which also remove the stop codon from the ubiquitin. Then the cDNA sequences of the antigens were amplified by PCR using the high fidelity Phusion HotStartII polymerase (FinnZymes, Thermo Fisher Scientific), subcloned in frame on the $3^{\prime}$ end of the ubiquitin sequence, using the same enzymes and a Rapid DNA ligation kit (Fermentas). All DNA vaccine plasmids were transformed into competent DH10b bacteria (Thermo Fisher Scientific) and Gigapreps were prepared using EndoFree Plasmid Giga Kit (Qiagen $\mathrm{AB}$ ) according to the manufacturer's protocol. Each plasmid batch was fully sequenced. Sequences of primers used for cloning and sequences of plasmids are available on request.

Vaccination. At time of vaccination, animals were anaesthetised with ketamine or telazol. In some animals, sedation was maintained via inhalational isoflurane as necessary. Vaccination was performed by disinfection of the injection site followed by an injection of $0.5-1 \mathrm{ml}$ of plasmid DNA diluted in saline to the quadriceps muscle. Immediately following instillation, the injection site was subject to electroporation by delivery of $0.6 \mathrm{~ms}^{2} 00 \mathrm{~V} \mathrm{~cm}^{-1}$ pulse followed by a $400 \mathrm{~ms}^{2} 0 \mathrm{~V} \mathrm{~cm}^{-1}$ pulse using a four-electrode array at a depth of $1 \mathrm{~cm}$. Electric pulses were delivered by the Genedrive device (IGEA). Animals were boosted at 3 -week intervals for a total of three vaccinations. Vaccinations were performed by trained personnel under supervision of veterinary staff. Animals were routinely monitored by veterinary staff, and detailed clinical exams performed weekly after vaccinations.

Virus. Animals were challenged with $1 \times 10^{5} \mathrm{TCID}_{50}$ of CCHFV strain Hoti divided between subcutaneous injections to the cranial dorsum and intravenously through the saphenous vein as previously described ${ }^{22}$. Our challenge stock of CCHFV Hoti was propagated, titered and sequenced as previously described ${ }^{6,22}$.

Interferon gamma ELISpot. PBMCs were isolated from EDTA-treated whole-blood spun over a Histopaque 1077 gradient (Sigma). Red blood cells were lysed with ACK lysis buffer (Gibco) and PBMCs frozen in fetal bovine serum supplemented with $10 \%$ dimethylsulfoxide (DMSO) (Hybrimax grade, Sigma) in liquid nitrogen vapour phase. Cryopreserved PBMCs were evaluated for IFN- $\gamma$ production in response to CCHFV-peptides by commercial ELISpot (Cellular Technologies Limited). PBMCs were thawed and plated at 100,000-400,000 cells per well in CTL-Test media. 15-mer peptides overlapping by 11 amino acids derived from the CCHFV NP or GPC were synthesized (Genscript), resuspended 
in DMSO (Hybrimax grade, Sigma) and pooled at $19-31$ peptides per pool. Cells were stimulated with peptide pools at a final concentration of $1 \mu \mathrm{g} \mathrm{ml}^{-1}$ each peptide. As positive control, cells were stimulated with concanavalin A (Life Technologies) or DMSO vehicle alone. Cells were incubated for $20 \mathrm{~h}$ at $37^{\circ} \mathrm{C}$ in $5 \% \mathrm{CO}_{2}$ before plates were developed according to the manufacturer's protocol. Spots were counted using an S6 Universal analyser (CTL) and data normalized per $1 \times 10^{6}$ cells. The upper limit of detection was set at 750 SFCs per well. Background was defined as number of spots in DMSO vehicle alone stimulated wells and this value subtracted from counts measured in peptide- and concanavalin A-stimulated wells. Each measurement was performed in duplicate.

ELISA. Whole-CCHFV-virion-specific IgM and IgG responses in serum were quantified by an in-house ELISA as previously described ${ }^{22}$. To measure NP or Gc-specific responses we performed a sandwich ELISA. Nunc Maxisorp plates were coated with $200 \mathrm{ng}$ per well of mouse monoclonal antibodies against NP (clone 9D5, BEI Resources) or Gc (11E7, BEI resources). Plates were then blocked with 5\% skim milk in PBS $0.05 \%$ Tween and then semi-purified whole virus lysate $^{22}$ applied. Plates were washed and a 1:1,000 dilution of serum applied. Bound antibodies were detected with goat horseradish peroxidase conjugated anti-monkey IgG (Seracare, catalogue no. KPL 074-11-021) at 1:2,000 and plates developed with ABTS solution (Seracare). Development was stopped with 5\% sodium dodecyl sulfate in water and absorbance at $405 \mathrm{~nm}$ read on a Synergy HTX (Biotek). For data analysis, absorbance values of wells coated with anti-NP or anti-Gc but receiving no CCHFV lysate were subtracted from wells receiving lysate. All measurements were performed in duplicate.

Serum neutralization titre. FRNTs were performed as previously described ${ }^{22,47}$.

RT-qPCR. Viral RNA in indicated tissues was quantified by RT-qPCR as previously described ${ }^{6}$.

Blood chemistry and haematology. Haematology was evaluated on EDTA-treated whole-blood using a Procyte DX (IDEXX Laboratories) and serum chemistries were completed on a Vetscan 2 (Abaxis) using Preventive care profile disks (Abaxis). Indicated normal ranges for blood parameters were obtained from published resources ${ }^{48,49}$

Immunohistochemistry. Immunohistochemistry to detect viral antigen was performed as described previously ${ }^{47}$.

Statistics and reproducibility. Indicated statistical tests were performed using Prism 8 (GraphPad). All data are derived from one experiment.

Reporting Summary. Further information on research design is available in the Nature Research Reporting Summary linked to this article.

\section{Data availability}

The data that support the findings of this study are available from the corresponding author upon reasonable request and source data for Figs. 1 and 3-5 are provided. Source data are provided with this paper.

Received: 14 May 2020; Accepted: 13 October 2020;

Published online: 30 November 2020

\section{References}

1. Bente, D. A. et al. Crimean-Congo hemorrhagic fever: history, epidemiology, pathogenesis, clinical syndrome and genetic diversity. Antiviral Res. 100, 159-189 (2013).

2. Negredo, A. et al. Autochthonous Crimean-Congo hemorrhagic fever in Spain. New Engl. J. Med. 377, 154-161 (2017)

3. Ergonul, O. Crimean-Congo haemorrhagic fever. Lancet Infect. Dis. https:// doi.org/10.1016/s1473-3099(06)70435-2 (2006).

4. Stuart, M. C., Kouimtzi, M. \& Hill, S. R. WHO Model Formulary 2008 (World Health Organization, 2008).

5. Johnson, S. et al. Ribavirin for treating Crimean Congo haemorrhagic fever. Cochrane Database Syst. Rev. 6, CD012713 (2018).

6. Hawman, D. W. et al. Favipiravir (T-705) but not ribavirin is effective against two distinct strains of Crimean-Congo hemorrhagic fever virus in mice. Antiviral Res. 157, 18-26 (2018).

7. Oestereich, L. et al. Evaluation of antiviral efficacy of ribavirin, arbidol, and T-705 (favipiravir) in a mouse model for Crimean-Congo hemorrhagic fever. PLoS Neglect. Trop. Dis. 8, e2804 (2014).

8. Bente, D. A. et al. Pathogenesis and immune response of Crimean-Congo hemorrhagic fever virus in a STAT-1 knockout mouse model. J. Virol. 84, 11089-11100 (2010)

9. Spengler, J. R., Bergeron, É. \& Rollin, P. E. Seroepidemiological studies of Crimean-Congo hemorrhagic fever virus in domestic and wild animals. PLoS Neglect. Trop. Dis. 10, e0004210 (2016).
10. Ergonul, O. et al. Systematic review and meta-analysis of postexposure prophylaxis for Crimean-Congo hemorrhagic fever virus among healthcare workers. Emerg. Infect. Dis. 24, 1642-1648 (2018).

11. Mousavi-Jazi, M., Karlberg, H., Papa, A., Christova, I. \& Mirazimi, A. Healthy individuals' immune response to the Bulgarian Crimean-Congo hemorrhagic fever virus vaccine. Vaccine 30, 6225-6229 (2012).

12. Papa, A., Papadimitriou, E. \& Christova, I. The Bulgarian vaccine Crimean-Congo haemorrhagic fever virus strain. Scandi. J. Infect. Dis. 43, 225-229 (2011)

13. Buttigieg, K. R. et al. A novel vaccine against Crimean-Congo haemorrhagic fever protects $100 \%$ of animals against lethal challenge in a mouse model PLOS ONE 9, e91516 (2014).

14. Zivcec, M., Safronetz, D., Scott, D. P., Robertson, S. \& Feldmann, H. Nucleocapsid protein-based vaccine provides protection in mice against lethal Crimean-Congo hemorrhagic fever virus challenge. PLoS Neglect. Trop. Dis. 12, e0006628 (2018).

15. Garrison, A. R. et al. A DNA vaccine for Crimean-Congo hemorrhagic fever protects against disease and death in two lethal mouse models. PLoS Neglect. Trop. Dis. 11, e0005908 (2017)

16. Ghiasi, S. M., Salmanian, A. H., Chinikar, S. \& Zakeri, S. Mice orally immunized with a transgenic plant expressing the glycoprotein of Crimean-Congo hemorrhagic fever virus. Clin. Vacc. Immunol. 18, 2031-2037 (2011).

17. Kortekaas, J. et al. Crimean-congo hemorrhagic fever virus subunit vaccines induce high levels of neutralizing antibodies but no protection in STAT1 knockout mice. Vector Borne Zoonotic Dis. 15, 759-764 (2015).

18. Canakoglu, N. et al. Immunization of knock-out $\alpha / \beta$ interferon receptor mice against high lethal dose of Crimean-Congo hemorrhagic fever virus with a cell culture based vaccine. PLoS Neglect. Trop. Dis. 9, e0003579 (2015).

19. Hinkula, J. et al. Immunization with DNA Plasmids Coding for Crimean-Congo Hemorrhagic Fever Virus Capsid and Envelope Proteins and/or Virus-Like Particles Induces Protection and Survival in Challenged Mice. J. Virol. 91, e02076-16 (2017).

20. Scholte, F. E. M. et al. Single-dose replicon particle vaccine provides complete protection against Crimean-Congo hemorrhagic fever virus in mice. Emerg. Microbes Infect. 8, 575-578 (2019).

21. Rodriguez, S. E. et al. Vesicular stomatitis virus-based vaccine protects mice against Crimean-Congo hemorrhagic fever. Sci. Rep. 9, 7755 (2019).

22. Haddock, E. et al. A cynomolgus macaque model for Crimean-Congo haemorrhagic fever. Nat. Microbiol. 3, 556-562 (2018).

23. Hawman, D. W. et al. Efficacy of favipiravir (T-705) against Crimean-Congo hemorrhagic fever virus infection in cynomolgus macaques. Antiviral Res. https://doi.org/10.1016/j.antiviral.2020.104858 (2020).

24. Kutzler, M. A. \& Weiner, D. B. DNA vaccines: ready for prime time? Nat. Rev. Genet. 9, 776-788 (2008).

25. Falzarano, D. et al. Treatment with interferon-alpha2b and ribavirin improves outcome in MERS-CoV-infected rhesus macaques. Nat. Med. 19, 1313-1317 (2013).

26. de Wit, E. et al. Prophylactic and therapeutic remdesivir (GS-5734) treatmen in the rhesus macaque model of MERS-CoV infection. Proc. Natl Acad. Sci. USA 117, 6771-6776 (2020).

27. Williamson, B. N. et al. Clinical benefit of remdesivir in rhesus macaques infected with SARS-CoV-2. Nature 585, 273-276 (2020).

28. Swanepoel, R. et al. The clinical pathology of Crimean-Congo hemorrhagic fever. Rev. Infect. Dis. 11, S794-S800 (1989).

29. Duh, D. et al. Viral load as predictor of Crimean-Congo hemorrhagic fever outcome. Emerg. Infect. Dis. 13, 1769-1772 (2007).

30. Hasanoglu, I. et al. Crucial parameter of the outcome in Crimean Congo hemorrhagic fever: viral load. J. Clin. Virol. 75, 42-46 (2016).

31. Ergonul, O., Celikbas, A., Baykam, N., Eren, S. \& Dokuzoguz, B. Analysis of risk-factors among patients with Crimean-Congo haemorrhagic fever virus infection: severity criteria revisited. Clin. Microbiol. Infect. 12, 551-554 (2006)

32. Aksoy, F., Yilmaz, G., Kaya, S., Karahan, S. C. \& Koksal, I. The prognostic importance of platelet indices in patients with Crimean-Congo hemorrhagic fever. Open Forum Infect. Dis. 4, S352-S353 (2017).

33. Çevik, M. A. et al. Clinical and laboratory features of Crimean-Congo hemorrhagic fever: predictors of fatality. Int. J. Infect. Dis. 12, 374-379 (2008).

34. Dowall, S. D. et al. Protective effects of a modified vaccinia Ankara-based vaccine candidate against Crimean-Congo haemorrhagic fever virus require both cellular and humoral responses. PLoS ONE 11, e0156637 (2016).

35. Bertolotti-Ciarlet, A. et al. Cellular localization and antigenic characterization of Crimean-Congo hemorrhagic fever virus glycoproteins. J. Virol. 79, 6152-6161 (2005)

36. Golden, J. W. et al. GP38-targeting monoclonal antibodies protect adult mice against lethal Crimean-Congo hemorrhagic fever virus infection. Sci. Adv. 5, eaaw9535 (2019)

37. Zivcec, M. et al. Identification of broadly neutralizing monoclonal antibodies against Crimean-Congo hemorrhagic fever virus. Antiviral Res. 146, $112-120$ (2017) 
38. Dowall, S. D. et al. A Crimean-Congo hemorrhagic fever (CCHF) viral vaccine expressing nucleoprotein is immunogenic but fails to confer protection against lethal disease. Hum. Vacc. Immunotherap. 12, 519-527 (2016).

39. Deyde, V. M., Khristova, M. L., Rollin, P. E., Ksiazek, T. G. \& Nichol, S. T Crimean-Congo hemorrhagic fever virus genomics and global diversity. J. Virol. https://doi.org/10.1128/jvi.00752-06 (2006).

40. André, F. \& Mir, L. M. DNA electrotransfer: its principles and an updated review of its therapeutic applications. Gene Ther. 11, S33-S42 (2004).

41. Weiland, O. et al. Therapeutic DNA vaccination using in vivo electroporation followed by standard of care therapy in patients with genotype 1 chronic hepatitis C. Mol. Ther. 21, 1796-1805 (2013).

42. Sardesai, N. Y. \& Weiner, D. B. Electroporation delivery of DNA vaccines: prospects for success. Curr. Opin. Immunol. 23, 421-429 (2011).

43. Zivcec, M., Spiropoulou, C. F. \& Spengler, J. R. The use of mice lacking type I or both type I and type II interferon responses in research on hemorrhagic fever viruses. Part 2: vaccine efficacy studies. Antiviral Res. 174, $104702(2020)$

44. Palacio, N. et al. Early type I IFN blockade improves the efficacy of viral vaccines. J. Exp. Med. https://doi.org/10.1084/jem.20191220 (2020).

45. Boshra, H., Lorenzo, G., Rodriguez, F. \& Brun, A. A DNA vaccine encoding ubiquitinated Rift Valley fever virus nucleoprotein provides consistent immunity and protects IFNAR ${ }^{-1-}$ mice upon lethal virus challenge. Vaccine 29, 4469-4475 (2011).

46. Rodriguez, F., Zhang, J. \& Whitton, J. L. DNA immunization: ubiquitination of a viral protein enhances cytotoxic T-lymphocyte induction and antiviral protection but abrogates antibody induction. J. Virol. 71, 8497 (1997).

47. Hawman, D. W. et al. A Crimean-Congo hemorrhagic fever mouse model recapitulating human convalescence. J. Virol. https://doi.org/10.1128/ JVI.00554-19 (2019).

48. Sasseville, V. G., Hotchkiss, C. E., Levesque, P. C. \& Mankowski, J. L. in Nonhuman Primates in Biomedical Research 2nd edn (eds Abee, C. R., Mansfield, K., Tardif, S. \& Morris, T.) 357-384 (Academic Press, 2012).

49. Choi, K. et al. Reference values of hematology, biochemistry, and blood type in cynomolgus monkeys from Cambodia origin. Lab. Animal Res. 32 46-55 (2016).

\section{Acknowledgements}

We thank the staff of the Rocky Mountain Veterinary Branch for their help with animal care and veterinary clinical and pathology work. Our work was funded and supported by the CCHVaccine consortium that has received funding from the European Union's Horizon 2020 research and innovation programme under grant agreement no. 732732 (A.M., M.S. and F.W.), by the Swedish Research Council (Vetenskapsrådet, V.R. and M.S.) section Research Environment, Infection Biology (contract number 2018-05766) (A.M. and F.W.), the Swedish Cancer Foundation (M.S.), the Stockholm County Council ALF and CIMED grants (M.S.), Vinnova CAMP grant (M.S.) and by the Intramural Research Program of the NIAID, NIH. The funders had no role in study design, data interpretation or decision to publish.

\section{Author contributions}

D.W.H., H.F., G.A., M.S., F.W. and A.M. conceived and designed experiments. D.W.H., G.A., K.S.A., K.M.-W., P.W.H., D.S., V.M. and S.D. performed experiments. K.S.A. and G.A. contributed equally. D.W.H., P.W.H., D.S., A.O., M.S., H.F. and A.M. analysed data. D.W.H., H.F., M.S. and A.M. prepared the manuscript. All authors discussed the results and manuscript, and agreed to final publication. H.F., M.S. and A.M. contributed equally as senior authors.

\section{Competing interests}

M.S. is a founder and chairman of the board of Svenska Vaccinfabriken Produktion AB.

\section{Additional information}

Extended data is available for this paper at https://doi.org/10.1038/s41564-020-00815-6. Supplementary information is available for this paper at https://doi.org/10.1038/ s41564-020-00815-6.

Correspondence and requests for materials should be addressed to A.M.

Reprints and permissions information is available at www.nature.com/reprints.

Publisher's note Springer Nature remains neutral with regard to jurisdictional claims in published maps and institutional affiliations.

(c) The Author(s), under exclusive licence to Springer Nature Limited 2020 
A

\begin{tabular}{|c|c|c|c|}
\hline Study number & DOB & Sex & Vaccine \\
\hline CCHF 61 & $1 / 26 / 2015$ & female & \\
\hline CCHF 62 & $3 / 5 / 2016$ & female & \multirow{2}{*}{ Sham } \\
\hline CCHF 63 & $2 / 5 / 2016$ & female & \\
\hline CCHF 64 & $2 / 11 / 2016$ & female & \\
\hline CCHF 65 & $1 / 18 / 2016$ & female & \\
\hline CCHF 66 & $1 / 10 / 2016$ & female & \\
\hline CCHF 67 & $1 / 10 / 2016$ & female & \\
\hline CCHF 68 & $11 / 12 / 2015$ & female & \\
\hline CCHF 69 & $2 / 5 / 2016$ & female & \\
\hline CCHF 70 & $2 / 11 / 2016$ & female & \\
\hline CCHF 71 & $2 / 22 / 2015$ & female & \\
\hline CCHF 72 & $2 / 9 / 2016$ & female & \\
\hline & & \\
\hline
\end{tabular}

$\mathrm{B}$

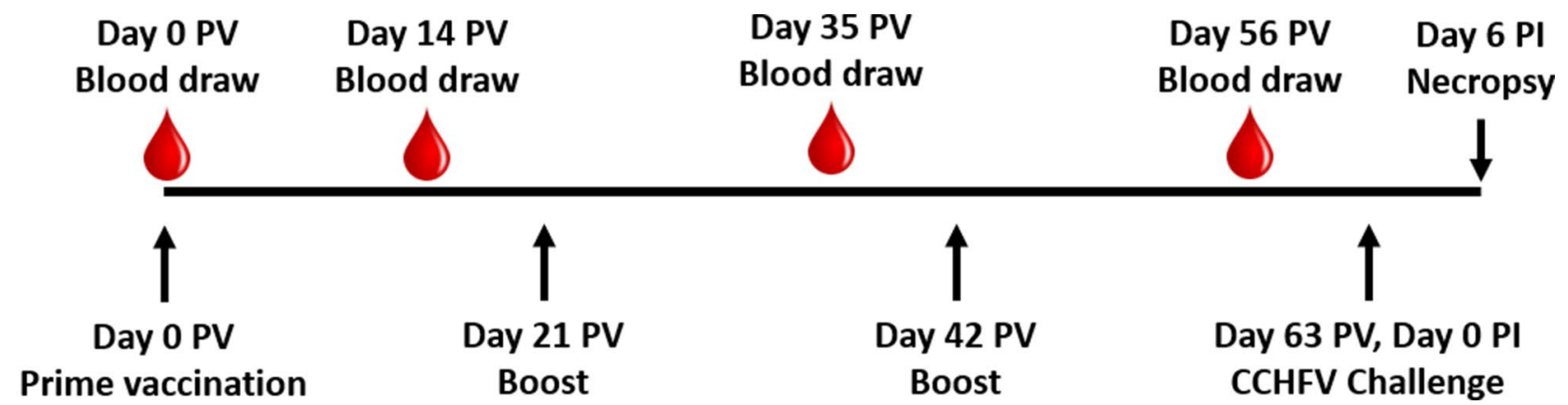

Extended Data Fig. 1 | Animal information and vaccination schedule. (a) Animal number, date of birth (DOB), sex and vaccination. Animals were randomly assigned to either group prior to start of study. (b) Vaccination, blood draw and CCHFV challenge schedule. PV = post prime-vaccination, $\mathrm{PI}=$ post-CCHFV infection. 
A

\begin{tabular}{|c|c|c|c|c|c|c|c|c|}
\hline \multicolumn{1}{|c|}{ Vaccination Site } & \multicolumn{3}{c|}{ Sham Vaccinated } & NP + GPC Vaccinate d \\
\hline $\begin{array}{c}\text { myonecrosis and loss with fibrosis and } \\
\text { histiocytosis with intracellular pigment }\end{array}$ & 3 & 2 & 1 & 1 & 0 & 1 & 1 & 0 \\
\hline
\end{tabular}
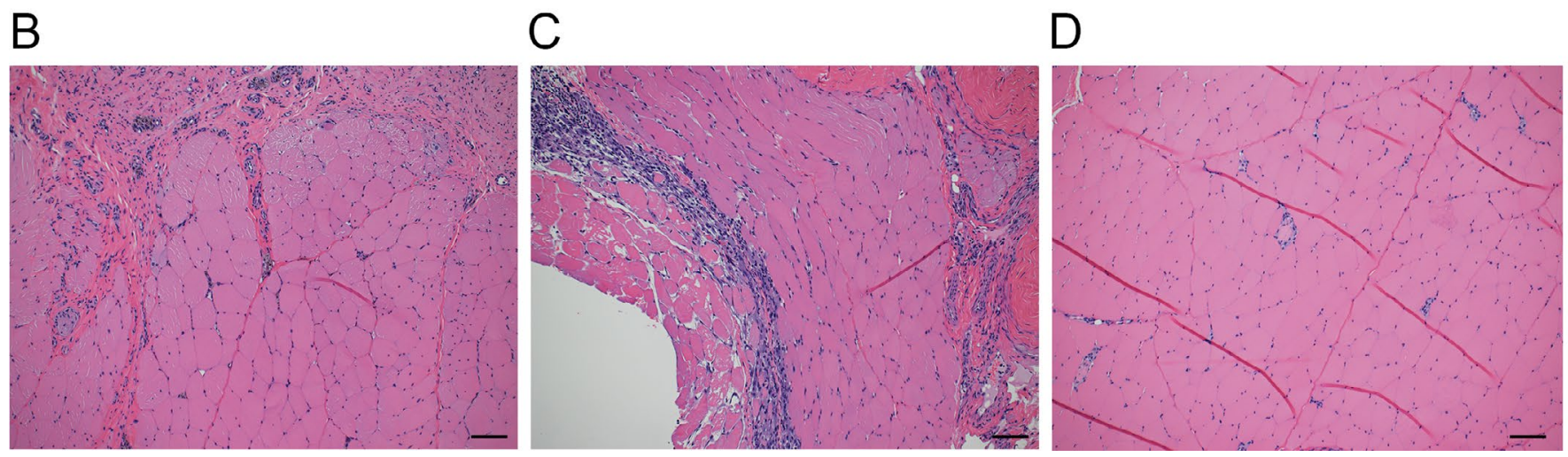

Extended Data Fig. 2 I Vaccination Site Histology. At Day +6 CCHFV challenge, 27 days after last vaccination, tissue from the vaccination site was fixed in $10 \%$ formalin and stained with hematoxylin and eosin. Slides were scored by a pathologist (a). $0=$ absent, $1=$ mild, $2=$ moderate, $3=$ severe. (b-d) Representative images of the one severe (b), one moderate (c) and one of six mild animals (d) are shown at 100x magnification. 


\section{Sham-Vaccinated IgG}

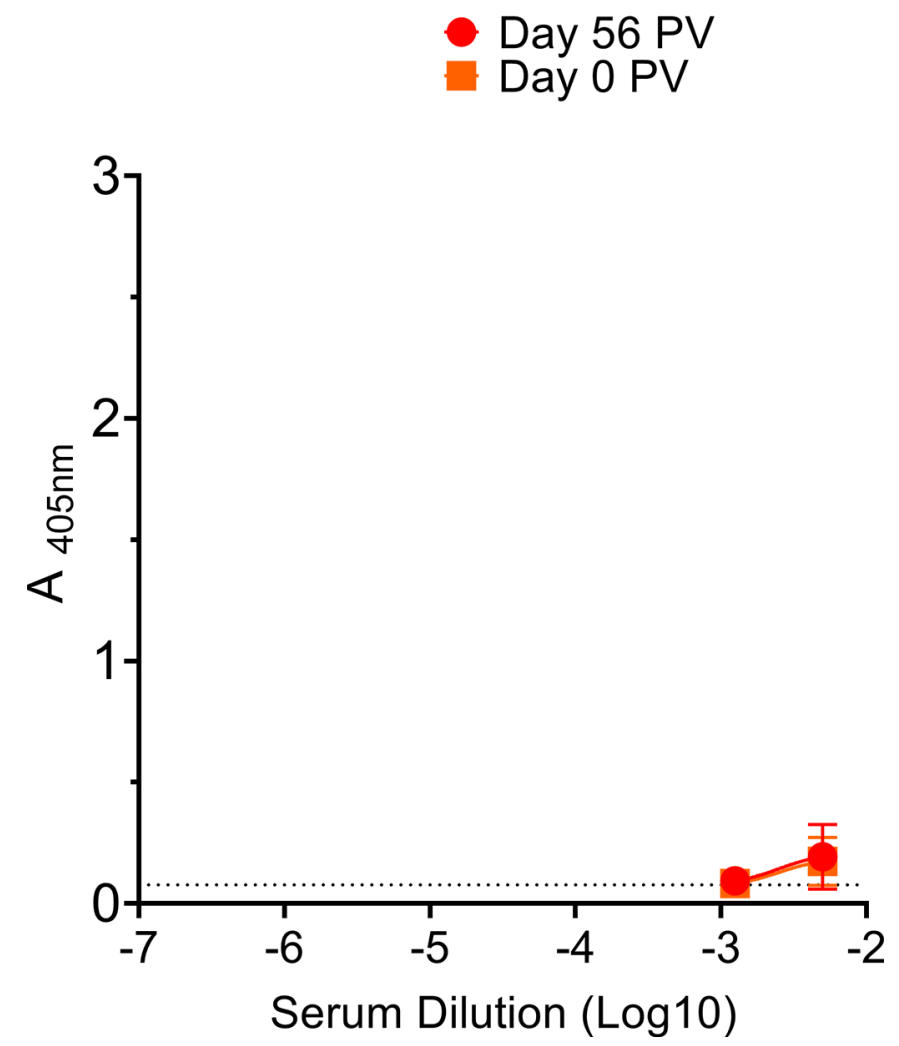

Extended Data Fig. 3 | CCHFV ELISA on serum from sham-vaccinated animals. At indicated time points, serum was collected from sham-vaccinated animals and CCHFV-specific IgG measured by ELISA on day 0 or 56 postprime vaccination. $\mathrm{N}=6$ animals per timepoint. Data shown as mean plus standard deviation. 


\section{Sandwich ELISA}

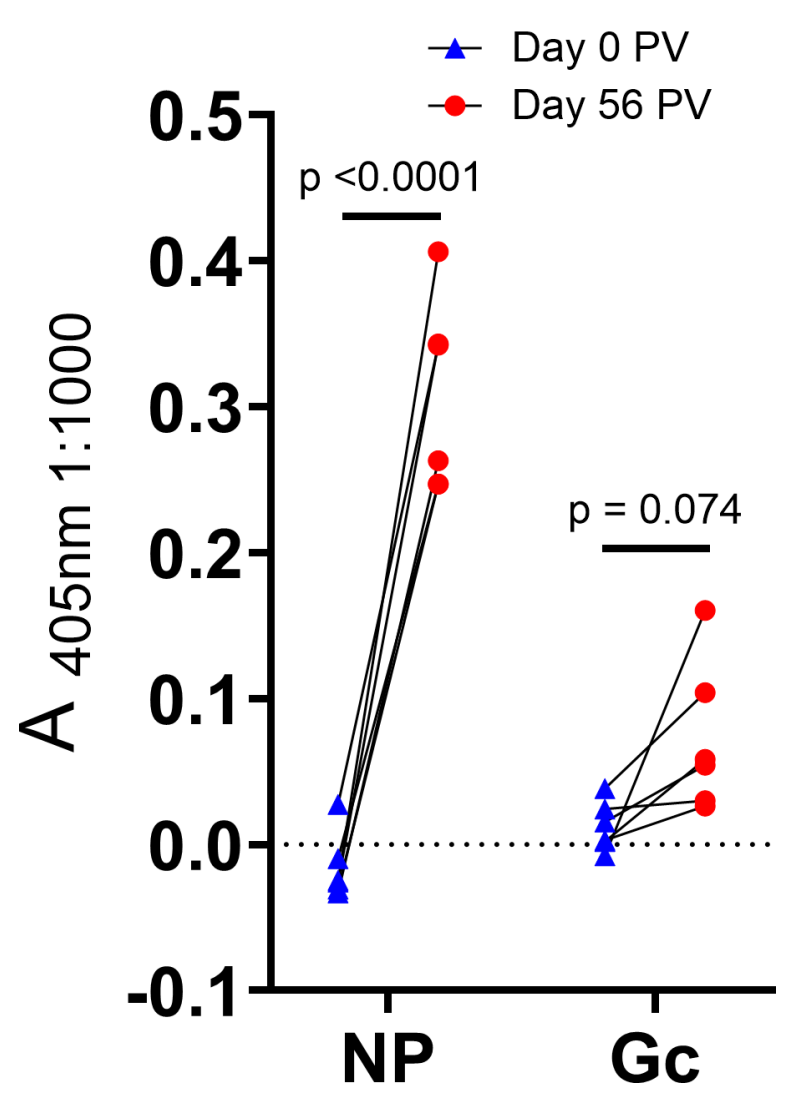

Extended Data Fig. 4 | Sandwich ELISA. A sandwich ELISA was performed on NP + GPC vaccinated animals to measure NP or Gc specific responses at day 0 post-prime vaccination (PV) and day 56 PV. N =6 animals per timepoint. Statistical comparison performed using a two-way ANOVA with Sidak's multiple comparison test. 
A
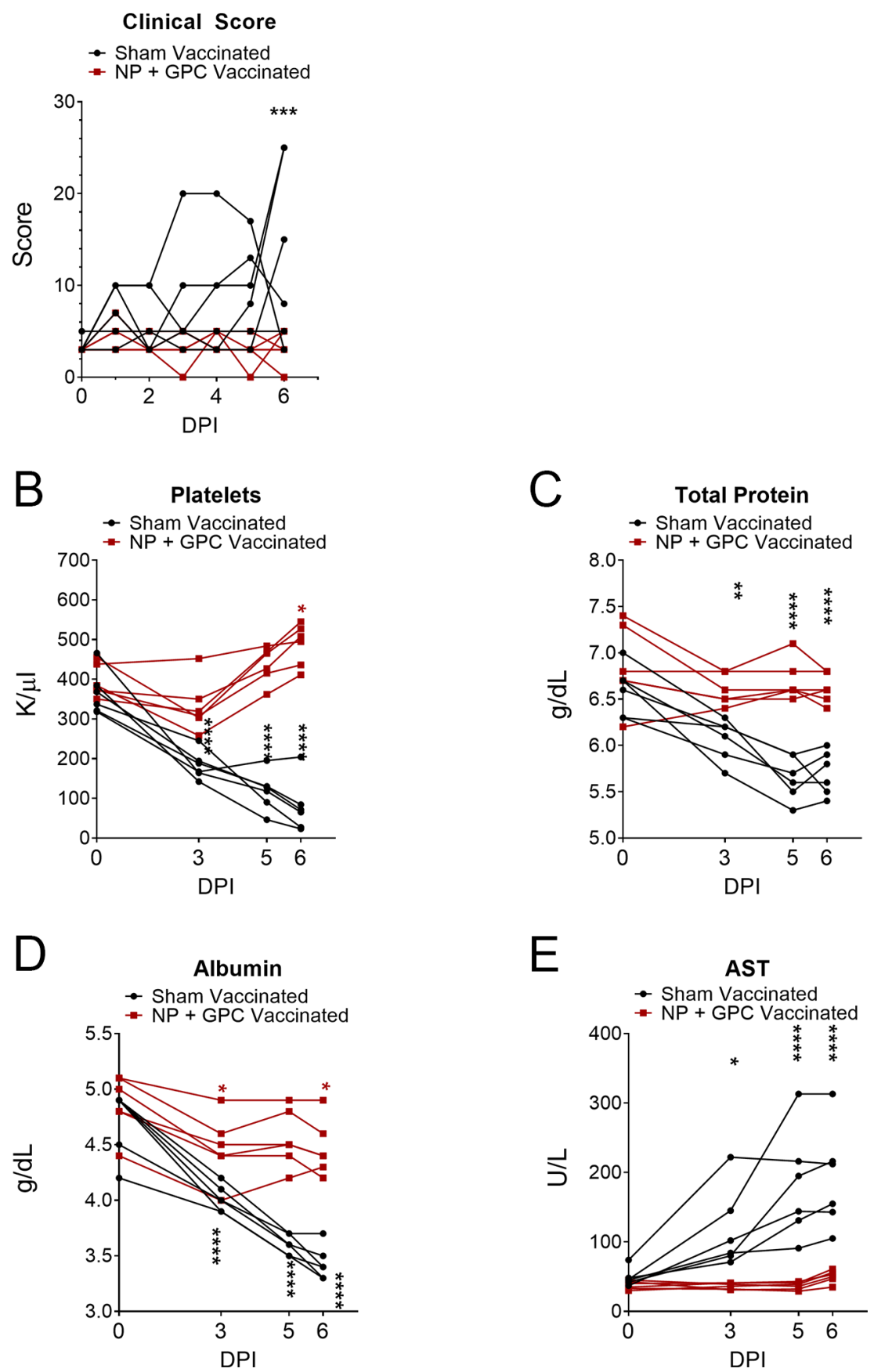

Extended Data Fig. 5 | Individual data for clinical scores and blood chemistry. The data from Fig. 3 is shown again but with individual data points shown. 


\section{Anamnestic Response}

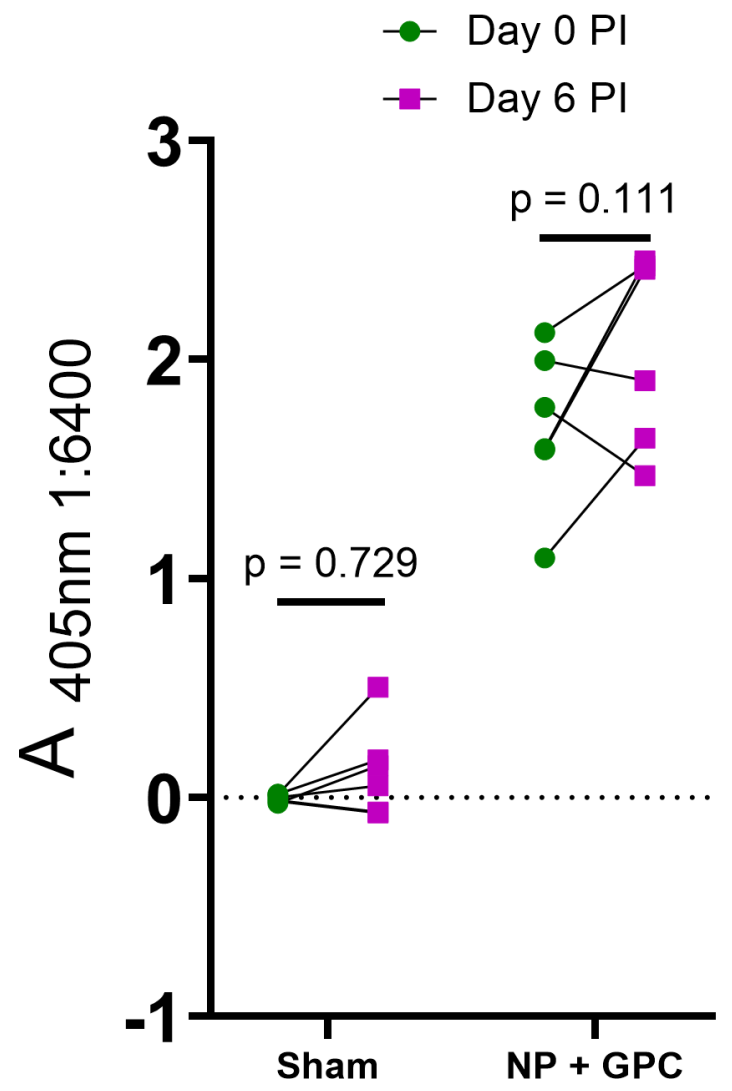

Extended Data Fig. 6 | Anamnestic response to CCHFV challenge. A whole-virion ELISA was used to measure antibody responses in animals after CCHFV challenge on day 6 post-infection (PI). CCHFV-specific IgG was quantified in a 1:6400 dilution of serum. Statistical tests performed using a two-way ANOVA with Sidak's multiple comparison test. 
ARTICLES

NATURE MICROBIOLOGY

Kazak $\quad$ Ub-G76A Linker

Antigen

stop

pVAX1-Ub-Antigen

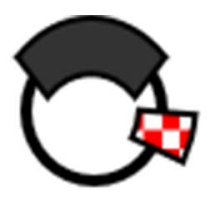

pVAX1-Ub

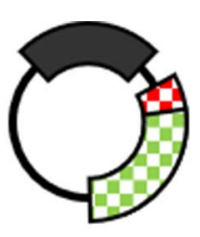

pVAX1-Ub_CCHFV-Hoti-N-CO

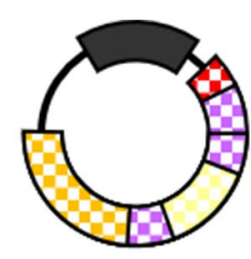

pVAX1-Ub_CCHFV-Hoti-M-CO

Extended Data Fig. 7 | General plasmid schematics of plasmids used in this study.

NATURE MICROBIOLOGY | www.nature.com/naturemicrobiology 


\section{Reporting Summary}

Nature Research wishes to improve the reproducibility of the work that we publish. This form provides structure for consistency and transparency in reporting. For further information on Nature Research policies, see our Editorial Policies and the Editorial Policy Checklist.

\section{Statistics}

For all statistical analyses, confirm that the following items are present in the figure legend, table legend, main text, or Methods section.

n/a Confirmed

$\square \bigotimes$ The exact sample size $(n)$ for each experimental group/condition, given as a discrete number and unit of measurement

Х $\square$ A statement on whether measurements were taken from distinct samples or whether the same sample was measured repeatedly

$\square$ The statistical test(s) used AND whether they are one- or two-sided

$\square$ Only common tests should be described solely by name; describe more complex techniques in the Methods section.

Х A description of all covariates tested

Х $\square$ A description of any assumptions or corrections, such as tests of normality and adjustment for multiple comparisons

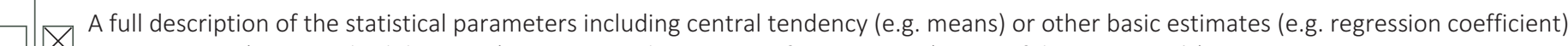

$\bigotimes$ AND variation (e.g. standard deviation) or associated estimates of uncertainty (e.g. confidence intervals)

$\square$ For null hypothesis testing, the test statistic (e.g. $F, t, r$ ) with confidence intervals, effect sizes, degrees of freedom and $P$ value noted

Give $P$ values as exact values whenever suitable.

Х $\square$ For Bayesian analysis, information on the choice of priors and Markov chain Monte Carlo settings

Х $\square$ For hierarchical and complex designs, identification of the appropriate level for tests and full reporting of outcomes

Х $\square$ Estimates of effect sizes (e.g. Cohen's $d$, Pearson's $r$ ), indicating how they were calculated

Our web collection on statistics for biologists contains articles on many of the points above.

\section{Software and code}

Policy information about availability of computer code

Data collection No software used for data collection

Data analysis Statistical analyses performed using Prism v8 (GraphPad)

For manuscripts utilizing custom algorithms or software that are central to the research but not yet described in published literature, software must be made available to editors and reviewers. We strongly encourage code deposition in a community repository (e.g. GitHub). See the Nature Research guidelines for submitting code \& software for further information.

\section{Data}

Policy information about availability of data

All manuscripts must include a data availability statement. This statement should provide the following information, where applicable:

- Accession codes, unique identifiers, or web links for publicly available datasets

- A list of figures that have associated raw data

- A description of any restrictions on data availability

The data that support the findings of this study are available from the corresponding

author upon reasonable request and source data for figures 1, 3,4 and 5 provided. 


\section{Field-specific reporting}

Please select the one below that is the best fit for your research. If you are not sure, read the appropriate sections before making your selection. $\bigotimes$ Life sciences $\quad \square$ Behavioural \& social sciences $\square$ Ecological, evolutionary \& environmental sciences

For a reference copy of the document with all sections, see nature.com/documents/nr-reporting-summary-flat.pdf

\section{Life sciences study design}

All studies must disclose on these points even when the disclosure is negative.

Sample size Group sizes were based upon reasonable estimates of the necessary number of animals needed to observe clinical benefit of the CCHFV vaccine. Similar group sizes have worked well in previous NHP studies

Data exclusions No data excluded from analyses.

Replication Due to ethical considerations of non-human primate research, studies were not repeated and were conducted once.

Randomization Animals were randomly assigned to CCHFV vaccination group or sham-vaccination group prior to study start.

Blinding Researchers were not blinded to groups. Due to constraints of working in ABSL4 and limitations of staff, it is not practical to blind researchers to groups

\section{Reporting for specific materials, systems and methods}

We require information from authors about some types of materials, experimental systems and methods used in many studies. Here, indicate whether each material, system or method listed is relevant to your study. If you are not sure if a list item applies to your research, read the appropriate section before selecting a response.

Materials \& experimental systems

\begin{tabular}{l|l}
\hline n/a & Involved in the study \\
$\square$ & $\square$ Antibodies \\
$\square$ & $\square$ Eukaryotic cell lines \\
$\square$ & $\square$ Clinical data \\
$\square$ & $\square$ Dual une research of concern
\end{tabular}

\section{Antibodies}

Antibodies used

anti-monkey IgG (Seracare, catalog KPL 074-11-021)

Validation

As per Seracare: "Affinity-purified polyclonal antibody to monkey IgG heavy chains (gamma), made in goat, and labeled with horseradish peroxidase. Product is in lyophilized form. Each lot is tested to assure specificity and lot-to-lot consistency using an inhouse ELISA assay."

\section{Animals and other organisms}

Policy information about studies involving animals; ARRIVE guidelines recommended for reporting animal research
Laboratory animals

Wild animals

Field-collected samples

Ethics oversight

\begin{tabular}{l|l} 
Methods \\
\hline n/a & Involved in the study \\
$\bigotimes$ & $\square$ ChIP-seq \\
$\bigotimes$ & $\square$ Flow cytometry \\
$\square$ & $\square$ MRI-based neuroimaging
\end{tabular}

Cynomolgus macaques, female, $>3$ years old

No wild animals used

No field samples collected

All animal work was performed in strict accordance with the recommendations described in the Guide for the Care and Use of Laboratory Animals of the Office of Animal Welfare, National Institutes of Health and the Animal Welfare Act of the US Department of Agriculture, in an Association for Assessment and Accreditation of Laboratory Animal Care International (AAALAC)-accredited facility.

Note that full information on the approval of the study protocol must also be provided in the manuscript. 